\title{
Generalized Koszul resolution
}

\author{
Satoshi Mochizuki and Akiyoshi Sannai
}

\begin{abstract}
The main objective of this paper is generalizing a notion of Koszul resolutions and charcterizing modules which admits such a resolution. We turn out that for a noetherian ring $A$ and a coherent $A$ module $M, M$ has a two dimensional generalized Koszul resolution if and only if $M$ is a pure weight two module in the sense of [HM09]. As its applications, we are attacking the Gersten conjecture for weight two case.
\end{abstract}

\section{Introduction}

In [Ger73] p.28, Gersten proposed the following conjecture

Conjecture 1.1 (Gersten's conjecture). For any commutative regular local ring $A$ and natural numbers $n, p$, the canonical inclusion $\mathcal{M}_{A}^{p+1} \hookrightarrow \mathcal{M}_{A}^{p}$ induces the zero map on $K$-groups

$$
\pi_{p}^{n}: K_{n}\left(\mathcal{M}_{A}^{p+1}\right) \rightarrow K_{n}\left(\mathcal{M}_{A}^{p}\right)
$$

where $\mathcal{M}_{A}^{i}$ is the category of finitely generated $A$-modules $M$ with

$\operatorname{Codim}_{\text {Spec } A} \operatorname{Supp} M \geqq i$.

In the case for $n=0$, as in the following proposition, Gersten's conjecture is equivalent to generator conjecture.

Proposition 1.2. (c.f. [Lev85][P.452, 1.1]) Let $A$ be a commutative regular local ring. Then the following statements are equivalent.

(i) The maps $K_{0}\left(\mathcal{M}_{A}^{p}\right) \rightarrow K_{0}\left(\mathcal{M}_{A}^{p-1}\right)$ are zero for $p=1, \cdots, \operatorname{dim} A$.

(ii) $K_{0}\left(\mathcal{M}^{p}(A)\right)$ is generated by cyclic modules $A /\left(f_{1}, \cdots, f_{p}\right)$ where $f_{1}, \cdots, f_{p}$ forms a regular sequence for $p=1, \cdots, \operatorname{dim} A$. 
One of the main consequence in this paper is deciding the generator of $K_{0}\left(\mathcal{M}_{A}^{2}\right)$ as follows.

Theorem 1.3 (Corollary 8.7). For a regular domain $A, K_{0}\left(\mathcal{M}_{A}^{2}\right)$ is generated by modules of a form $\frac{A^{\oplus n}}{<\operatorname{Im} P, \operatorname{Im} Q>}$ where $P$ and $Q$ are endomorphisms on $A^{\oplus n}$ such that $P$ and $Q$ are similar to the following type matrixes:

$$
\begin{gathered}
P \sim\left(\begin{array}{cc}
f E_{m} & 0 \\
0 & E_{n-m}
\end{array}\right), \\
Q \sim\left(\begin{array}{cc}
g E_{m} & 0 \\
0 & E_{n-m}
\end{array}\right)
\end{gathered}
$$

where $f, g$ forms a regular sequences and $E_{k}$ is the $k$-th unit matrix. Moreover if $P$ and $Q$ are commutative, then the class of $\frac{A^{\oplus n}}{\langle\operatorname{Im} P, \operatorname{Im} Q>}$ is in the kernel of $\pi_{2}^{0}$.

For non-regular $A$, we have similar statement by replacing with $K_{0}\left(\mathcal{M}_{A}^{2}\right)$ by the Grothendieck group of perfect complexes. (see 8.7).

The main point of this paper is to deal with such problem with new theory of complexes and resolutions. More precisely, the theory of generalized Koszul resolutions and related this notion with pure weight modules defined in [HM09] and Koszul cubes defined in [Kos]. To state the main theorem now we define the notion of generalized Koszul resolution and Koszul cubes.

Definition 1.4 (Generalized Koszul resolutions). A $n$-cube $x$ in a category $\mathcal{C}$ is a contravariant functor from $[1]^{\times n}$ to $\mathcal{C}$ where $[1]$ is a totally ordered set $\{0,1\}$ with the natural order $0<1$. For each $\mathfrak{i} \in[1]^{\times n}$, we call $x(\mathfrak{i})$ a vertex of $x$. For each $\mathfrak{i}=\left(i_{1}, \cdots, i_{n}\right)$, we write $x\left(\mathfrak{i}-\mathfrak{e}_{k} \rightarrow \mathfrak{i}\right)$ by $d_{\mathfrak{i}}^{k}$ where $\mathfrak{e}_{k}$ is the $k$-th unit vector and we assume that $i_{k}=1$.

Let $f_{1}, \cdots, f_{n}$ be a regular sequence in $A$.

A Koszul cube associated with $f_{1}, \cdots, f_{n}$ is a $n$-cube $x$ in $\mathcal{M}_{A}\left(=\mathcal{M}_{A}^{0}\right)$ satisfying the following conditions:

(1) each vertex of $x$ is a finite free $A$-modules and their rank is constant. Therefore we can consider the determinant of its boundary maps.

(2) there are positive integers $m_{1}, \cdots, m_{n}$ and $\operatorname{det} d_{\mathfrak{i}}^{k}=f_{k}^{m_{s}}$ for each $\mathfrak{i}=$ $\left(i_{1}, \cdots, i_{n}\right) \in[1]^{\times n}$ and $1 \leqq k \leqq n$ such that $i_{k}=1$.

A generalized Koszul resolution associated with $f_{1}, \cdots, f_{n}$ is the totalized complex of a Koszul cubes associated with $f_{1}, \cdots, f_{n}$. 
Now we state the main theorems:

Theorem 1.5 (Theorem 7.1). For any $M$ in $\mathcal{M}_{A}$ and a regular sequence $f$, $g$, the following conditions are equivalent:

(1) $M$ is pure weight two modules supported on $V(f, g)$ in the sense of [HM09].

(2) $M$ is resolved by a generalized Koszul resolution associated with $f, g$.

(3) There is a Koszul cube $x$ associated with $f, g$ such that $\mathrm{H}_{0}(\operatorname{Tot} x)$ is isomorphic to $M$.

Finally the second author propose the conjecture 8.9 about purely linear algebra which implies Gersten's conjecture for $K_{0}$-groups for three dimensional regular local rings.

Conventions. Throughout of this paper, we use the letter $A$ to denote a commutative noethrian ring with a unit. We denote the category of finitely generated $A$-modules, the category of finitely generated $A$-modules by $\mathcal{M}_{A}$, $\mathcal{P}_{A}$ respectively.

Acknowledgements. The second author is grateful for Kei-ichi Watanabe and Takafumi Shibuta for stimulating discussion.

\section{Definition of weight}

In this section, we start from reviewing a notion of pure weight perfect modules over noetherian rings. For more information of pure weight perfect modules over any schemes, see [HM09]. Mainly we intend to study fundamental properties of pure weight modules over a Cohen-Macaulay local ring.

Definition 2.1. For an ideal $I$ of $A$ generated by a regular sequence of $A$ and $0 \leqq r \leqq \infty$, let us denote the category of finitely generated $A$-modules of projective dimension $\leqq r$ supported on $V(I)$ by $\mathcal{M}_{A}^{I}(r)$. One can easily check that $\mathcal{M}_{A}^{I}(r)$ is closed under the extensions and direct summand in $\mathcal{M}_{A}$. Therefore $\mathcal{M}_{A}^{I}(r)$ has a natural exact categorical structure. If $I$ is generated by a regular sequence consisting of $r$ elements, we denote $\mathcal{M}_{A}^{I}(r)$ by $\mathbf{W t}_{A}^{I}$ and an $A$-module in $\mathbf{W t}_{A}^{I}$ is said to be pure weight $r$ A-module (supported on $I$ ). 
Remark 2.2. The definition above is compatible with that in [HM09]. To prove this we shall notice that the notion of torsion and projective dimension are equivalent for a finitely generated modules on a noetherian ring (see [Wei94, Proposition 4.1.5]) and that in the notation above, $\operatorname{Spec} A / I \hookrightarrow \operatorname{Spec} A$ is a regular closed immersion.

Example 2.3. Let $f_{1}, \cdots, f_{p}$ be a regular sequence of $A$, then $A /\left(f_{1}, \ldots, f_{p}\right)$ is a typical example of a module of weight $p$. We will call such a pure weight module a simple pure weight module.

A module of weight 0 is a projective module whose support is total space $\operatorname{Spec} A$.

If $A$ is a Cohen-Macaulay local ring of Krull dimension $d$, a module of weight $d$ is just a module of finite projective dimension and finite length.

If $A$ is not a Cohen-Macaulay ring, the class of $A$-modules of finite length and finite projective dimension does not work fine as in the following example in [Ger74]. Let $(A, \mathfrak{m})$ be a 2-dimensional local ring which is not normal and Spec $A \backslash\{\mathfrak{m}\}$ is regular. Then an $A$-Module of finite length and finite projective dimension is the zero $A$-module.

Rough sketch of proof. Let $M$ be a non-zero, $A$-module of finite length and finite projective dimension. Since $A$ is not Cohen-Macaulay, we have inequalities

$$
\operatorname{Projdim}_{A} M \leqq \operatorname{Projdim}_{A} M+\operatorname{depth}_{A} M=\operatorname{depth} A<2 .
$$

Let

$$
A^{\oplus n} \stackrel{\phi}{\rightarrow} A^{\oplus m} \rightarrow M \rightarrow 0
$$

be a projective $(=$ free) resolution of $M$. Then we can easily notice that $n=m$ and let us put $f=\operatorname{det} \phi$ and let $\mathfrak{p}$ be one of minimal prime of the ideal $(f)$. Then by Krull's theorem, we have ht $\mathfrak{p}=1$ and we can easily notice that $M_{\mathfrak{p}} \neq 0$. But by hypothesis we have $\operatorname{Supp} M=\{\mathfrak{m}\}$. This is contradiction.

In this section, from now on, we assume that $A$ is a Cohen-Macaulay ring.

Notations 2.4. Let us denote the category of weight $p A$-modules by $\mathbf{W t}_{A}^{p}$. Since $\mathbf{W} \mathbf{t}_{A}^{p}$ is closed under extensions in $\mathcal{M}_{A}, \mathbf{W t}_{A}^{p}$ can be naturally considered as an exact category.

In this section from now on, we moreover assume that $A$ is local ring of Krull dimension $d$ 
Proposition 2.5. Let $M$ be a non-zero, pure weight $p$ A-module. Then $M$ is a Cohen-Macaulay module of dimension $d-p$.

Proof. We have two equalities:

$$
\begin{gathered}
\operatorname{dim}_{A} M+\operatorname{Codim}_{A} M=d \\
\operatorname{Projdim}_{A} M+\operatorname{depth}_{A} M=d
\end{gathered}
$$

Therefore we have

$$
d-p \leqq \operatorname{depth}_{A} M \leqq \operatorname{dim}_{A} M \leqq d-p .
$$

Hence we get $\operatorname{depth}_{A} M=\operatorname{dim}_{A} M=d-p$.

Corollary 2.6. For a non-zero pure weight module $M$, its associated prime ideal is minimal.

Proof. It is a general property of Cohen-Macaulay modules.

\section{Generality of cubes}

In this section, we fix a general notion of cubes. From now on, let $\mathcal{C}$ be a category.

Notations 3.1. For a set $S$, we denote its power set by $\mathcal{P}(S)$. That is, $\mathcal{P}(S)$ is the set of all subset of $S$. By the natural inclusion order, we can consider $\mathcal{P}(S)$ as an order sets, a fortiori, a category. For a map $f: S \rightarrow T$ between sets, we define its push forward which is an order preserving map as follows.

$$
f_{*}: \mathcal{P}(S) \ni x \rightarrow f(x) \in \mathcal{P}(T) .
$$

Obviously the association above is functorial, we get the functor

$$
\mathcal{P}: \text { Set } \rightarrow \text { Cat }
$$

where Set, Cat are the category of sets, the category of categories respectively. 
Example 3.2. For a positive integer $n$, we denote the set of positive integers $k$ such that $1 \leqq k \leqq n$ by $(n]$ and for a non-negative integer $m$, we denote the totally order set of integers $k$ such that $0 \leqq k \leqq n$ with the natural order by $[n]$. We put $\mathcal{P}_{n}=\mathcal{P}((n])$. Then we have the canonical category isomorphism

$$
\begin{gathered}
\mathcal{P}_{n} \stackrel{\sim}{\rightarrow}[1]^{\times n} \\
S \mapsto\left(\chi_{S}(1), \cdots, \chi_{S}(n)\right)
\end{gathered}
$$

where $\chi_{S}$ is the characteristic function associated with $S$, that is, the function $\chi_{S}:(n] \rightarrow[1]$ defined by

$$
\chi_{S}(k)=\left\{\begin{array}{ll}
1 & \text { if } k \in S \\
0 & \text { otherwise }
\end{array} .\right.
$$

Example 3.3. For a pair of disjoint sets $S, T$, we have the canonical category equivalence:

$$
\mathcal{P}(S) \times \mathcal{P}(T) \ni(A, B) \mapsto A \cup B \in \mathcal{P}(S \sqcup T) .
$$

Definition 3.4. For a set $S$, a $S$-cube in $\mathcal{C}$ is a contravariant functor from $\mathcal{P}(S)$ to $\mathcal{C}$. We denote the category of $S$-cubes in $\mathcal{C}$ by

$$
\operatorname{Cub}^{S}(\mathcal{C}):=\mathcal{H O} \mathcal{M}\left(\mathcal{P}(S)^{\text {op }}, \mathcal{C}\right)
$$

Since the association $\mathcal{H O} \mathcal{M}\left(\mathcal{P}(S)^{\text {op }}\right.$, ?) is covariant, we get the functors

$$
\begin{gathered}
\mathrm{Cub}^{S}: \text { Cat } \rightarrow \text { Cat } \\
\text { Cub : Set } \ni S \mapsto \mathbf{C u b}^{S} \in \mathcal{E N} \mathcal{D}(\text { Cat }):=\mathcal{H O} \mathcal{M}(\text { Cat, Cat }) .
\end{gathered}
$$

Notations 3.5. Let $S$ be a set and $x$ be a $S$-cube in $\mathcal{C}$. For $T \in \mathcal{P}(S)$ and $k \in T$, we denote $x(S)$ by $x_{S}$ and call it a vertex of $x$. we also write $x(T \backslash\{k\} \hookrightarrow T)$ by $d_{T}^{x, k}$ or shortly $d_{T}^{k}$ and call it a boundary morphism of $x$.

The following lemma is sometimes useful to treat morphisms of cubes.

Lemma 3.6. Let $S$ be a set.

(1) For any $S$-cube $x$, every $T, U \in \mathcal{P}(S)$ such that $T \subset U$ and $U \backslash T$ is a finite set, $x(U \subset T)$ is described as composition of boundary morphisms.

(2) Assume that $S$ is a finite set. For any $S$ cubes $x, y$ and a family of morphisms $f=\left\{f_{T}: x_{T} \rightarrow y_{T}\right\}_{T \in \mathcal{P}(S)}$ in $\mathcal{C}, f: x \rightarrow y$ is a morphism of $S$-cubes in $\mathcal{C}$ if and only if for any $T \in \mathcal{P}(S)$ and $k \in T$, we have the equality $d_{T}^{y, k} f_{T}=f_{T \backslash\{k\}} d_{T}^{x, k}$. 
Example 3.7. Let $S$ be a set.

(1) If $S$ is the emptyset $\emptyset$, then we have the canonical isomorphism of endofunctors on Cat:

$$
\mathrm{Cub}^{\emptyset} \stackrel{\sim}{\rightarrow} \operatorname{id}_{\text {Cat }}
$$

(2) We denote the morphism category of $\mathcal{C}$ is the category whose objects are morphisms in $\mathcal{C}$ and morphisms are commutative squares in $\mathcal{C}$ by $\operatorname{Ar} \mathcal{C}$. The assignment $\mathcal{C} \mapsto \operatorname{Ar} \mathcal{C}$ above is functorial, therefore we have the endofunctor:

$$
\text { Ar : Cat } \rightarrow \text { Cat }
$$

We have the canonical two natural transformations

$$
\text { dom, ran }: \text { Ar } \rightarrow \text { id }_{\text {Cat }} \text {. }
$$

Namely, for any morphism $f: x \rightarrow y$ in $\mathcal{C}$, we have $\operatorname{dom}(f)=x$ and $\operatorname{ran}(f)=$ $y$.

If $S$ is a singleton $\{s\}$, then we have the canonical isomorhpsim of endofunctors on Cat:

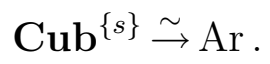

Notations 3.8. For a positive integer $n,(n]$-cubes are simply said to be $n$-cubes and $\mathbf{C u b}^{(n]}$ is denoted by $\mathbf{C u b}{ }^{n}$. By the isomorphism in 3.2, we sometimes consider $n$-cubes as contravariant functors on $[1]^{\times n}$. Then for a $n$-cube $x$ in $\mathcal{C}$, we denote $x\left(i_{1}, \cdots, i_{n}\right)$ by $x_{i_{1}, \cdots, i_{n}}$ and $x\left(\left(i_{1}, \cdots, \hat{1}, \cdots, i_{p}\right) \rightarrow\right.$ $\left.\left(i_{1}, \cdots, \hat{0}, \cdots, i_{p}\right)\right)$ by $d_{\dot{i}_{1}, \cdots, j_{j}, \cdot, i_{n}}^{x, j}$ or shortly $d_{\dot{i}_{1}, \cdots,,_{j}, \cdot, i_{n}}^{j} \cdot$

Example 3.9. For a pair of disjoint sets $S, T$, a $S$-cube in $T$-cube is canonically considered as a $S \sqcup T$-cube. More precisely, utilizing the isomorphism in 3.3, we have the canonical isomorphism between endfunctors on Cat

$$
\mathrm{Cub}^{S \sqcup T} \stackrel{\sim}{\rightarrow} \mathrm{Cub}^{S}\left(\mathrm{Cub}^{T}\right) .
$$

Example 3.10. Let $f_{1}, \cdots, f_{n}$ be elements in $A$. We define the $n$-cube $\operatorname{Kos}\left(f_{1}, \cdots, f_{n}\right)$ (or shortly $\left.\operatorname{Kos}\left(\mathfrak{f}_{1}^{n}\right)\right)$ in $\mathcal{P}_{A}$ by $\operatorname{Kos}\left(\mathfrak{f}_{1}^{n}\right)_{S}:=A$ for any $S \in \mathcal{P}_{n}$ and $d_{S}^{j}=f_{j}$ for any $S \in \mathcal{P}_{n}$ and $j \in S$. 
Notations 3.11. For a set $S$ and an element $k \in S$, we have the two kind of canonical natural transformations $\delta_{S, \text { dom }}^{k}, \delta_{S, \text { ran }}^{k}: \mathbf{C u b}^{S} \rightarrow \mathbf{C u b}^{S \backslash\{k\}}$ defined by the composition

$$
\operatorname{Cub}^{S}(\mathcal{C}) \stackrel{\sim}{\rightarrow} \operatorname{Cub}^{\{k\}}\left(\mathrm{Cub}^{S \backslash\{k\}}(\mathcal{C})\right) \stackrel{\sim}{\rightarrow} \operatorname{Ar}\left(\operatorname{Cub}^{S \backslash\{k\}}(\mathcal{C})\right) \rightarrow \operatorname{Cub}^{S \backslash\{k\}}(\mathcal{C})
$$

where the first morphism is defined in 3.9, the second one is described in 3.7 and the last one is dom or ran respectively. For a $S$-cube $x$ in $\mathcal{C}, \delta_{S, \text { dom }}^{k}(x)$, $\delta_{S, \text { ran }}^{k}(x)$ are said to be the domain side $k$-face, range side $k$-face of $x$ respectively.

The following lemma is sometimes useful for treating cubes.

Lemma 3.12 (Cube lemma). For the diagram in the category $\mathcal{C}$ :

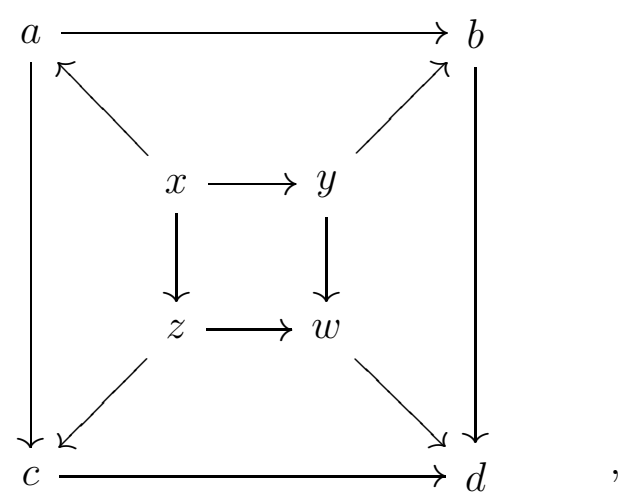

assume that the morphism $\overrightarrow{w d}$ is a monomorphism (resp. $\overrightarrow{x a}$ is an epimorphism) and every squares except xywz (resp. abdc) are commutative. Then xywz (resp. abdc) is also commutative.

From now on, we assume that $\mathcal{C}$ has a zero object.

Definition 3.13 (Homology of cubes). Let $S$ be a set, $x$ a $S$-cube in $\mathcal{C}$ and an element $k$ in $S$.

Assume that for any $T \in \mathcal{P}(S \backslash\{k\})$, there exist a cokernel (resp. a kernel) of $d_{T \cup\{k\}}^{k}$, then the $k$-direction 0 -th (resp. 1-th) homology of $x$ is a $S \backslash\{k\}$-cube in $\mathcal{C}$ denoted by $\mathrm{H}_{0}^{k}(x)$ (resp. $\left.\mathrm{H}_{1}^{k}(x)\right)$ and defined by $\mathrm{H}_{0}^{k}(x)_{T}:=$ Coker $d_{T \cup\{k\}}^{k}$ $\left(\right.$ resp. $\left.\mathrm{H}_{1}^{k}(x)_{T}:=\operatorname{ker} d_{T \cup\{k\}}^{k}\right)$.

There are the natural quotient and inclusion morphisms $H_{1}^{k}(x) \hookrightarrow x_{T \cup\{k\}}$ and 
$x_{T} \rightarrow H_{0}^{k}(x)$. The associations above are functrial, that is, if we assume that any morphism in $\mathcal{C}$ admits its cokernel (resp. kernel), then we have the following functor

$$
\mathrm{H}_{0}^{k}\left(\operatorname{resp} . \mathrm{H}_{1}^{k}\right): \mathbf{C u b}^{S}(\mathcal{C}) \rightarrow \mathbf{C u b}^{S \backslash\{k\}}(\mathcal{C})
$$

and the natural transformations

$$
\mathrm{H}_{1}^{k} \stackrel{\iota_{S}^{k}}{\rightarrow} \delta_{S, \text { dom }}^{k} \text { and } \delta_{S, \text { ran }}^{k} \stackrel{\pi_{S}^{k}}{\rightarrow} \mathrm{H}_{0}^{k}
$$

\section{Notations for multi complexes}

Now we are fixing the notation of multi complexes in an additive category. We assume that $\mathcal{A}$ is an additive category.

Notations 4.1. For a set $S$, let us denote the set of maps from $S$ to $\mathbb{Z}$ by $\mathbb{Z}^{S}$. It has the natural term-wise addition, that is, for any $f, g$ in $\mathbb{Z}^{S}$, we define $f+g$ by $(f+g)(s):=f(s)+g(s)$ for any $s$ in $S$. Let us recall that for any $T \in \mathcal{P}(S), \chi_{T} \in \mathbb{Z}^{S}$ is the characteristic function associated with $T$ (see 3.2). In particular for any $s \in S, \chi_{\{s\}}$ is denoted by $\delta^{s}$.

Definition 4.2 (Multi complexes). For a set $S$, a $S$-multi complexes $x_{\bullet}$ in $\mathcal{A}$ is a family $\left\{x_{f}, d_{f}^{x, s}\right\}_{f \in \mathbb{Z}^{S}, s \in S}$ consisting of objects $x_{f}$ in $\mathcal{C}$ and morphisms $d_{f}^{x, s}: x_{f} \rightarrow x_{f-\delta^{s}}$ satisfying the following equalities:

$$
\left\{\begin{array}{l}
d_{f-\delta^{s}}^{x, s} d_{f}^{x, s}=0 \\
d_{f-\delta^{s}}^{x, t} d_{f}^{x, s}+d_{f-\delta^{t}}^{x, s} d_{f}^{x, t}=0
\end{array}\right.
$$

for every $f \in \mathbb{Z}^{S}$ and $s \neq t$ in $S$.

For each $f \in \mathbb{Z}^{S}$ and $s \in S, d_{f}^{x, s}$ is said to be boundary map and sometimes written by $d_{f}^{s}$ shortly.

A $S$-multi complex $x_{\bullet}$ is bounded if except finitely many $f \in \mathbb{Z}^{S}, x_{f}=0$.

A morphism of $S$-multi complexes $\phi_{\bullet}: x_{\bullet} \rightarrow y_{\bullet}$ is a family of morphisms $\left\{\phi_{f}: x_{f} \rightarrow y_{f}\right\}_{f \in \mathbb{Z}^{S}}$ satisfying the equality:

$$
d_{f}^{y, s} \phi_{f}=\phi_{f-\delta^{s}} d_{f}^{x, s}
$$

for any $f \in \mathbb{Z}^{S}$ and $s$ in $S$. Let us denote the category of $S$-multi complexes on $\mathcal{A}$ by $\mathbf{C h}^{S}(\mathcal{A})$ and the category of bounded $S$-multi complexes on $\mathcal{A}$ by 
$\mathrm{Ch}_{b}^{S}(\mathcal{A})$

Therefore we get the functors

$$
\mathrm{Ch}^{S}, \mathrm{Ch}_{b}^{S}: \text { AddCat } \rightarrow \text { AddCat }
$$

where AddCat is the category of additive categories.

Example 4.3. For the empty set $\emptyset, \mathbf{C h}^{\phi}(\mathcal{A})=\mathcal{A}$ and for an one element set $\{s\}$, we write $\mathbf{C h}^{\{s\}}$ by $\mathbf{C h}$. $\mathbf{C h}(\mathcal{A})$ is the usual category of chain complexes in $\mathcal{A}$ by identifying $\mathbb{Z}^{\{s\}}=\mathbb{Z}$. In this case, for a complex in $\operatorname{Ch}(\mathcal{A})$, we simply denote its boundary maps $d_{n}^{x, s}$ by $d_{n}^{x}$ or shorty $d_{n}$.

Remark 4.4 (Sign notations). For a non-negative integer $n$, we have the canonical isomorphism of functors

$$
\mathbf{C h}^{(n]} \stackrel{\sim}{\rightarrow} \mathbf{C h}^{n}
$$

where $\mathbf{C h}^{n}$ is the $n$-times iteration of the functor $\mathbf{C h}$. For the $(n]$-multi complex $x_{\bullet}$ in $\mathcal{A}$, we put $d_{f}^{\prime k}:=(-1)^{\sum_{t=k+1}^{n} f(t)} d_{f}^{k}$ for each $f \in \mathbb{Z}^{(n]}$ and $k \in$ $(n]$. Then one can easily verify $\left\{x_{f}, d_{f}^{\prime k}\right\}$ is an object in $\mathbf{C h}^{n}(\mathcal{A})$. This correspondence gives the isomorphism above. By the identification above, we say the $(n]$-multi complexes as the $n$-multi complexes.

the $n$-th cubes in $\mathcal{A}$ is also naturally considered as the $n$-th multi complexes in $\mathcal{A}$.

Example 4.5. For a pair of disjoint finite sets $S, T$, by utilizing the isomorphisms in 4.4, we have a non-canonical isomorphism of functors

$$
\begin{aligned}
\mathbf{C h}^{S \sqcup T} \stackrel{\sim}{\rightarrow} \mathbf{C h}^{S}\left(\mathbf{C h}^{T}\right), \\
\mathbf{C h}_{b}^{S \sqcup T} \stackrel{\sim}{\rightarrow} \mathbf{C h}_{b}^{S}\left(\mathbf{C h}_{b}^{T}\right) .
\end{aligned}
$$

Definition 4.6 (Total functors). For a finite set $S$, we define the total functor

$$
\operatorname{Tot}^{S}: \mathbf{C h}_{b}^{S} \rightarrow \mathbf{C h}_{b}
$$

as follows. $\operatorname{Tot}^{S}(x)_{n}:=\bigoplus_{\sum_{k \in S} f(k)=n} x_{f}$ and $d_{n}:=\sum_{\sum_{k \in S} \in S} d_{f(k)=n}^{s}$.

For a pair of disjoint finite sets $S$ and $T$, we fix isomorphisms in 4.5,

$$
\alpha: \mathbf{C h}_{b}^{S \sqcup T} \stackrel{\sim}{\rightarrow} \mathbf{C h}_{b}^{S}\left(\mathbf{C h}_{b}^{T}\right),
$$




$$
\beta: \mathbf{C h}_{b}\left(\mathbf{C h}_{b}^{T}\right) \stackrel{\sim}{\rightarrow} \mathbf{C h}^{T \sqcup\{*\}}
$$

where $*$ is a symbol which is not in $T$. Then we have the functor $\operatorname{Tot}_{\alpha, \beta}^{S}$ : $\mathbf{C h}^{S \sqcup T} \rightarrow \mathbf{C h}^{T \sqcup\{*\}}$ by the composition of functors below:

$$
\mathbf{C h}_{b}^{S \sqcup T} \stackrel{\alpha}{\rightarrow} \mathbf{C h}_{b}^{S}\left(\mathbf{C h}_{b}^{T}\right) \stackrel{\text { Tot }^{S}}{\rightarrow} \mathbf{C h}_{b}\left(\mathbf{C h}_{b}^{T}\right) \stackrel{\beta}{\rightarrow} \mathbf{C h}^{T \sqcup\{*\}} .
$$

We often omit $\alpha$ and $\beta$ in the notation.

\section{Admissible cubes}

In this section, we will study a specific class of cubes, so called, admissible cubes on an abelian category. From now on, let us fix an abelian category $\mathcal{A}$ and $S$ a finite set. We start from considering a typical example.

Definition 5.1. A regular sequence $f_{1}, \cdots, f_{n}$ is $A$-sequence if $f_{\sigma(1)}, \cdots, f_{\sigma(n)}$ is also regular sequence for any bijection $\sigma$ on $(n]$.

Remark 5.2. For any regular sequence $f_{1}, \cdots, f_{n}$, if the condition (a) or (b) below is satisfied, then $f_{1}, \cdots, f_{n}$ is an $A$-sequence.

(a) $f_{1}, \cdots, f_{n}$ is contained in the Jacobson radical in $A$.

(b) For each $i, A /\left(f_{1}, \cdots, f_{i}\right) A$ is complete for $\left(f_{1}, \cdots, f_{n}\right)$-adic topology.

Example 5.3. For a sequence $f_{1}, \cdots, f_{n}$ in $A$, it is $A$-sequence if and only if for any $k$ in $[n-1]$ and distinct numbers $i_{1}, \cdots, i_{k}$ in $(n]$, a map $f_{i_{k+1}}: A /\left(f_{i_{1}}, \cdots, f_{i_{k}}\right) \rightarrow A /\left(f_{i_{1}}, \cdots, f_{i_{k}}\right)$ is injective. This is equivalent to the $n$-cube $\operatorname{Kos}\left(\mathfrak{f}_{1}^{n}\right)$ (for definition, see 3.10) satisfies the following condition: For any $k$ in $[n-1]$ and distinct numbers $i_{1}, \cdots, i_{k}$ in $(n]$, boundary maps of $\mathrm{H}_{0}^{i_{1}}\left(\cdots\left(\mathrm{H}_{0}^{i_{k}}\left(\operatorname{Kos}\left(\mathfrak{f}_{1}^{n}\right)\right)\right) \cdots\right)$ are injections.

In the case, $\operatorname{Kos}\left(\mathfrak{f}_{1}^{n}\right)$ is said to be the typical Koszul cubes associated with $f_{1}, \cdots, f_{n}$.

Definition 5.4 (Admissible cubes). Let $x$ be a $S$-cube. If $\sharp S=1, x$ is called admissible if its boundary morphism is a monomorphism. Inductively, for $\sharp S>1, x$ is called admissible if its boundary morphisms are monomorphism and if for every $k$ in $S, \mathrm{H}_{0}^{k}(x)$ is admissible.

Lemma 5.5. For an admissible $S$-cube $x$ in $\mathcal{A}$ and distinct elements $i_{1}, \cdots, i_{k}$ in $S$, we have the canonical isomorphism:

$$
\mathrm{H}_{0}^{i_{1}}\left(\mathrm{H}_{0}^{i_{2}}\left(\cdots\left(\mathrm{H}_{0}^{i_{k}}(x)\right) \cdots\right)\right) \stackrel{\sim}{\rightarrow} \mathrm{H}_{0}^{i_{\sigma}(1)}\left(\mathrm{H}_{0}^{i_{\sigma}(2)}\left(\cdots\left(\mathrm{H}_{0}^{i_{\sigma}(k)}(x)\right) \cdots\right)\right)
$$

where $\sigma$ is a bijection on $S$. 
Proof. It is enough to prove the case for $S=\{1,2\}$. Then by $3 \times 3$-lemma, we turn out that $\mathrm{H}^{2}\left(\mathrm{H}^{1}(x)\right)$ and $\mathrm{H}^{1}\left(\mathrm{H}^{2}(x)\right)$ is canonical isomorphic to the object $y$ in the diagram below.

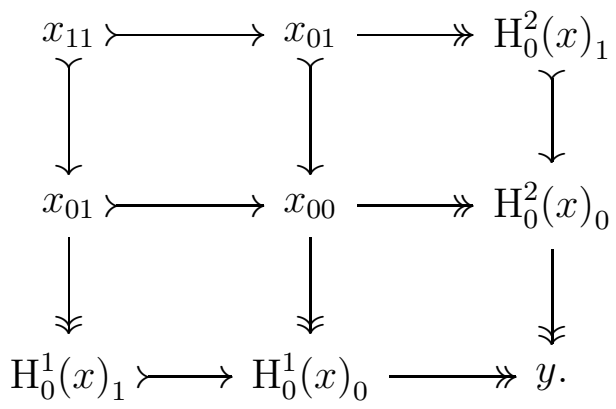

Notations 5.6. For an admissible $S$-cube $x$ in $\mathcal{A}$ and a subset $T=\left\{i_{1}, \cdots, i_{k}\right\} \subset$ $S$, we put $\mathrm{H}_{0}^{T}(x):=\mathrm{H}_{0}^{i_{1}}\left(\mathrm{H}_{0}^{i_{2}}\left(\cdots\left(\mathrm{H}_{0}^{i_{k}}(x)\right) \cdots\right)\right)$.

Proposition 5.7. Let $x$ be a $S$-cube in $\mathcal{A}$. Assume that every $k$-face of $x$ is admissible, then for any $k$ in $S$, we have

$$
\mathrm{H}_{p}\left(\operatorname{Tot}^{S}(x)\right) \stackrel{\sim}{\rightarrow}\left\{\begin{array}{ll}
\mathrm{H}_{p}\left(\mathrm{H}_{0}^{S \backslash\{k\}}(x)\right) & \text { for } p=0,1 \\
0 & \text { otherwise }
\end{array} .\right.
$$

In particular if we assume that $x$ is admissible, then we have

$$
\mathrm{H}_{p}\left(\operatorname{Tot}^{S}(x)\right)=\left\{\begin{array}{ll}
\mathrm{H}_{0}^{S}(x) & \text { for } p=0 \\
0 & \text { otherwise }
\end{array} .\right.
$$

Proof. We intend to use induction for the number of elements in $S$. For $\sharp S=1$, the assertion is trival. Now let us consider for $n \sharp S>1$. For simplicity, we put $T=S \backslash\{k\}$. Let us consider the spectral sequence associated with the bi-complex $\operatorname{Tot}^{T}(x)$,

$$
\mathrm{H}_{p}^{v} \mathrm{H}_{q}^{h}\left(\operatorname{Tot}^{T}(x)\right) \Rightarrow \mathrm{H}_{p+q}\left(\operatorname{Tot}^{S}(x)\right) .
$$

Considering the short exact sequences associated with the spectral sequence above

$$
0 \rightarrow \mathrm{H}_{0}^{v} \mathrm{H}_{q}^{h}\left(\operatorname{Tot}^{T}(x)\right) \rightarrow \mathrm{H}_{q}\left(\operatorname{Tot}^{S}(x)\right) \rightarrow \mathrm{H}_{1}^{v} \mathrm{H}_{q-1}^{h}\left(\operatorname{Tot}^{T}(x)\right) \rightarrow 0,
$$


and noticing that by inductive hypothesis, we have the isomorphisms

$$
\mathrm{H}_{q}^{h}\left(\operatorname{Tot}^{T}(x)\right) \stackrel{\sim}{\rightarrow}\left\{\begin{array}{ll}
\mathrm{H}_{0}^{T}(x) & \text { for } q=0 \\
0 & \text { otherwise }
\end{array},\right.
$$

we get the isomorphisms

$$
\mathrm{H}_{p}\left(\operatorname{Tot}^{S}(x)\right) \stackrel{\sim}{\rightarrow}\left\{\begin{array}{ll}
\mathrm{H}_{p}^{v}\left(\mathrm{H}_{0}^{T}(x)\right) & \text { for } p=0,1 \\
0 & \text { otherwise }
\end{array} .\right.
$$

Hence we obtain the assertion.

\section{Generalized Koszul resolution}

In this section, we introduce a generalization of Koszul resolution. Let us start from reviewing Koszul complexes.

Review 6.1 (Koszul complex). Let $f: P \rightarrow Q$ be a $A$-module homomorphism between projective $A$-modules. The $n$-th Koszul complex associated with $f$ is denoted by $\operatorname{Kos}^{n}(f)$ and defined as follows:

$$
\operatorname{Kos}^{n}(f)_{k}=\Lambda^{k}(P) \otimes \operatorname{Sym}^{n-k}(Q)
$$

and the Koszul differential $d_{k+1}: \operatorname{Kos}^{n}(f)_{k+1} \rightarrow \operatorname{Kos}^{n}(f)_{k}$ is given by

$p_{1} \wedge \cdots \wedge p_{k+1} \otimes q_{k+2} \cdots q_{n} \mapsto \sum_{i=1}^{k+1}(-1)^{k+1-i} p_{1} \wedge \cdots \wedge \hat{p}_{i} \wedge \cdots \wedge p_{k+1} \otimes f\left(p_{i}\right) q_{k+2} \cdots q_{n}$.

Example 6.2. Let $f_{1}, \cdots, f_{n}$ be elements in $A$. We consider a homomorphism $\mathfrak{f}=\left(\begin{array}{lll}f_{1} & \cdots & f_{n}\end{array}\right): A^{\oplus n} \rightarrow A$. For simplicity, the $n$-th Koszul complex associated with $\mathfrak{f}$ is denoted by $\operatorname{Kos}(\mathfrak{f})$. Notice that $\operatorname{Tot}^{(n]}\left(\operatorname{Kos}\left(\mathfrak{f}_{1}^{n}\right)\right)$, the total complex of $\operatorname{Kos}\left(\mathfrak{f}_{1}^{n}\right)$ defined in 3.10, is isomorphic to $\operatorname{Kos}(\mathfrak{f})$ above.

It is well-known that if $f_{1}, \cdots, f_{n}$ forms regular sequence, then $\mathrm{H}_{k}(\operatorname{Kos}(\mathfrak{f}))$ is trivial for $k \geqq 1$. In this section, we intend to generalize this fact.

Definition 6.3 (Generalized Koszul complexes). Let $n, m$ be positive integers and $f_{1}, \cdots, f_{n}$ elements in $A$. For a family of endomorphisms on $A^{\oplus m}$ $\mathfrak{d}=\left\{d_{S}^{j}\right\}_{S \in \mathcal{P}_{n}, j \in S}$ such that $\operatorname{det} d_{S}^{j}=f_{j}$ for any $S \in \mathcal{P}_{n}$ and $j \in S$, we define 
the generalized Koszul complex associated with $\mathfrak{d}, \operatorname{Kos}(\mathfrak{d})$ as follows.

$\operatorname{Kos}(\mathfrak{d})_{k}:=\bigoplus_{\substack{S \in \mathcal{P}_{n} \\ \sharp S=k}} F_{S}$ where $F_{S}:=A^{\oplus m}$ and its boundary maps are defined by

$$
(-1)^{\sum^{t=j+1} \chi_{S}(t)} d_{S}^{j}: F_{S} \rightarrow F_{S \backslash\{j\}}
$$

on its $F_{S}$ component.

Remark 6.4. The definition in 6.3 is equivalent to that $\operatorname{Kos}(\mathfrak{d})$ is the total complex of the following $n$-cube $x$ :

$x_{S}:=A^{\oplus m}$ and $d_{S}^{s}:=d_{S}^{s}$ for any $S \in \mathcal{P}_{n}$ and $s \in S$.

Therefore we also denote $x$ by $\operatorname{Kos}(\mathfrak{d})$.

Let us recall that a complex $E_{\text {e }}$ on an abelian category is said to be $n$-spherical if $\mathrm{H}_{k}\left(E_{\bullet}\right)=0$ unless $k \neq n$.

Theorem 6.5. For a family $\mathfrak{d}$ as in the notation 6.3 if $f_{1}, \cdots, f_{n}$ forms a regular sequence, $\operatorname{Kos}(\mathfrak{d})$ is 0-spherical.

To prove the theorem above, we need to use Buchsbaum and Eisenbud Theorem. To state the theorem, we start from recalling the notation of ideal of minors.

Definition 6.6 (Ideal of minors). Let $U$ be an $m \times n$ matrix over $A$ where $m, n$ are positive integers. For $t$ in $(\min (m, n)]$ we then denote by $I_{t}(U)$ the ideal generated by the $t$-minors of $U$, that is, the determinant of $t \times t$ sub-matrices of $U$.

For an $A$-module homomorphism $\phi: M \rightarrow N$ between finite free $A$-modules, let us choose a matrix representation $U$ with respect to bases of $M$ and $N$. One can easily prove that the ideal $I_{t}(U)$ only depend on $\phi$. Therefore we put $I_{t}(\phi):=I_{t}(U)$.

Theorem 6.7 (Buchsbaum-Eisenbud [BE73]). For a complex of finite free A-modules

$$
F_{\bullet}: 0 \rightarrow F_{s} \stackrel{\phi_{s}}{\longrightarrow} F_{s-1} \stackrel{\phi_{s-1}}{\rightarrow} \rightarrow \cdots \rightarrow F_{1} \stackrel{\phi_{1}}{\rightarrow} F_{0} \rightarrow 0
$$

set $r_{i}=\sum_{j=i}^{s}(-1)^{j-i} \operatorname{rank} F_{j}$. Then the following are equivalent:

(1) $F_{\bullet}$ is 0-spherical.

(2) grade $I_{r_{i}}\left(\phi_{i}\right) \geqq i$ for any $i$ in $(s]$. 
Proof of Theorem 6.5. We denote boundary maps of $\operatorname{Kos}(\mathfrak{d})$ and $\operatorname{Kos}\left(\mathfrak{f}_{1}^{n}\right)$ by $d_{k}^{\mathfrak{d}}$ and $d_{k}^{\mathfrak{f}}$ respectively. We also put

$$
r_{i}=\sum_{j=i}^{n}(-1)^{j-i} \operatorname{rank} \operatorname{Kos}\left(\mathfrak{f}_{1}^{n}\right)_{j}=\sum_{j=i}^{n}(-1)^{j-i}\left(\begin{array}{l}
n \\
j
\end{array}\right) .
$$

Then we have

$$
\sum_{j=i}^{n}(-1)^{j-i} \operatorname{rank} \operatorname{Kos}\left(\mathfrak{d}_{1}^{n}\right)_{j}=m r_{i}
$$

In the notation 6.3 if $m=1$, then $\operatorname{Kos}(\mathfrak{d})=\operatorname{Kos}(\mathfrak{f})$ and in this case, the assertion is well-known. Therefore by 6.7 , it follows that grade $I_{r_{i}}\left(d_{i}^{\mathfrak{f}}\right) \geqq i$ for any $i$ in $(n]$. But inspection shows that for each $i \in(n], I_{r_{i}}\left(d_{i}^{\mathfrak{f}}\right)=$ $I_{n r_{i}}\left(d_{i}^{\mathfrak{d}}\right)$. Therefore we use Theorem 6.7 again, we turn out that $\operatorname{Kos}(\mathfrak{d})$ is 0 -spherical.

Corollary 6.8. For a family $\mathfrak{d}$ as in the notation 6.3 , if $f_{1}, \cdots, f_{n}$ forms A-sequences, then the cube $\operatorname{Kos}(\mathfrak{d})$ is admissible.

Proof. We prove by induction for $n$. In the case for $n=1$, the assertion is trivial. For $n>1$, by inductive hypothesis, we notice that all $k$-faces of $\operatorname{Kos}(\mathfrak{d})$ is admissible for $k \in(n]$. For each $k$, then by 5.7 , we have the isomorphism:

$$
\mathrm{H}_{1}\left(\mathrm{H}_{0}^{(n] \backslash\{k\}}(\operatorname{Kos}(\mathfrak{d}))\right) \stackrel{\sim}{\rightarrow} \mathrm{H}_{1}\left(\operatorname{Tot}^{(n]}(\operatorname{Kos}(\mathfrak{d}))\right)
$$

and by 6.5 , we turn out that the groups above is trivial. It means that $\operatorname{Kos}(\mathfrak{d})$ is admissible.

Definition 6.9 (Generalized Koszul resolutions). Let $f_{1}, \cdots, f_{n}$ be an $A$ sequence.

A Koszul $(n$ - $)$ cube associated with $f_{1}, \cdots, f_{n}$ is a $n$-cube $x$ in $\mathcal{M}_{A}$ satisfying the following conditions:

(1) each vertex of $x$ is a finite free $A$-modules and their rank is constant. Therefore we can consider the determinant of its boundary maps.

(2) there are positive integers $m_{1}, \cdots, m_{n}$ and $\operatorname{det} d_{S}^{s}=f_{s}^{m_{s}}$ for each $S \in \mathcal{P}_{n}$ and $s \in S$.

A generalized Koszul resolution associated with $f_{1}, \cdots, f_{n}$ is the totalized complex of a Koszul cubes associated with $f_{1}, \cdots, f_{n}$. Let us denote the category of Koszul cubes (resp. generalized Koszul resolution) associated with $f_{1}, \cdots, f_{n}$ by $\operatorname{Kos}_{A}^{f_{1}^{n}}$ (resp. $\operatorname{GKos}_{A}^{f_{1}^{n}}$ ). 
Notations 6.10 (Rank and determinants of Koszul cubes). For an $n$-Koszul cube $x$, we define $\operatorname{rank}$ of $x$ by $\operatorname{rank} x:=\operatorname{rank} x_{\emptyset}$. We also define $j$-th determinant of $x$ by $\operatorname{det}_{j} x:=\operatorname{det} d_{\{j\}}^{j}$ for any $j \in(n]$.

Remark 6.11. By 6.5, a generalized Koszul resolution is 0 -spherical and by 6.8 , Koszul cube is an admissible cubes.

As in the notation 6.9, we have the totalized functor

$$
\text { Tot : } \operatorname{Kos}_{A}^{\mathfrak{f}_{1}^{n}} \rightarrow \operatorname{GKos}_{A}^{\mathfrak{f}_{1}^{n}}
$$

which is essentially surjective and faithful.

Remark 6.12 (Compatibility of the definition in [Kos]). Now let us assume that $A$ is a noetherian ring such that every finite projective $A$-module is free. For example, the $n$-th valuable polynomial ring over a principle ideal domain or local ring and so on.

Then in [Kos], the notion of Koszul cubes is defined as follows:

A Koszul $n$-cube $x$ associated with an $A$-sequence $f_{1}, \cdots, f_{n}$ is an admissible $n$-cube in $\mathcal{M}_{A}$ which satisfies the following conditions.

(1) each $X_{S}$ is free $A$-modules for any $S \in \mathcal{P}_{n}$.

(2) For each $i \in(n]$, the vertexes of $\mathrm{H}_{0}^{k}(x)$ are in $\mathbf{W} \mathbf{t}_{A}^{\left(f_{i}\right)}$.

Utilizing the lemma 6.13 below, we turn out that the definition 6.9 and the definition above is equivalent if we assume that all $f_{i}$ is a prime element.

Lemma 6.13. Let $f$ be a non-zero divisor. For an A-module homomorphism

$$
\psi: A^{\oplus n} \rightarrow A^{\oplus n}
$$

Let us consider the following assertions.

(1) $\operatorname{det} \psi=f^{\alpha} \times$ unit for some $\alpha$.

(2) Coker $\psi$ is in $\mathbf{W t}_{A}^{(f)}$ and $\psi$ is injective.

The assertion (1) implies the assertion (2). Moreover if we assume that $f$ is a prime element, then the assertion (2) implies the assertion (1).

Proof. First we assume that the assertion (1). There is the cofactor morphism $\tilde{\psi}$ of $\psi$ such that $\tilde{\psi} \psi$ is multiplication by $f^{\alpha} \times$ unit. Hence $\psi$ is injective and Coker $\psi$ has projective dimension one. Since $\psi_{f}$ is an isomorphism, we turn out that Coker $\psi$ is supported on $V(f)$. Now we assume the assertion (2) and that $f$ is a prime element, then we notice that $\psi_{f}$ is an isomorphism and it means that $\operatorname{det} \psi$ is in $A_{f}^{\times}$. Now we turn out that $\operatorname{det} \psi=f^{\alpha} \times$ unit for some $\alpha$. 


\section{Weight two cases}

In this section, we assume that $A$ is noetherian domain and let $f, g$ be an $A$-sequence. First we give a characterization of weight two modules.

Theorem 7.1. For any $M$ in $\mathcal{M}_{A}$, the following conditions are equivalent.

(1) $M$ is in $\mathbf{W t}_{A}^{(f, g)}$.

(2) $M$ is resolved by a generalized Koszul resolution associated with $f, g$.

(3) There is a Koszul cube $x$ in $\mathbf{K o s}_{A}^{f, g}$ such that $\mathrm{H}_{0}($ Tot $x)$ is isomorphic to $M$.

Obviously the assertion that (2) implies (1) and that (3) implies (2) are trivial.

Proof. Let us fix an $A$-module $M$ in $\mathbf{W} \mathbf{t}_{A}^{(f, g)}$. By replacing $f$ with $f^{\alpha}$ for some $\alpha$ and so on, without loss of generality, we may assume that $f M=g M=0$. Then now we have a surjection $(A / f)^{\oplus n} \rightarrow M$ with kernel $L$. Since $\mathbf{W t}_{A}^{(f)}$ is closed under taking kernels of surjections, $L$ is in $\mathbf{W t} \mathbf{t}_{A}^{(f)}$. By considering resolutions of $L$ and $(A / f)^{\oplus n}$, we get the following diagram.

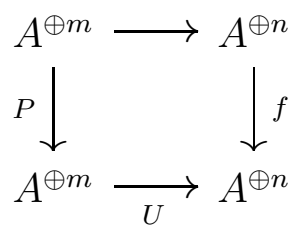

Claim $\operatorname{det} P=$ unit $\times f^{n}$.

Proof of Claim. First Localize at $f$ for the sequence below

$$
A^{\oplus m} \stackrel{P}{\rightarrow} A^{\oplus m} \rightarrow L \rightarrow 0
$$

we notice that $\frac{\operatorname{det} p}{f^{n}}$ is in $A_{f}^{\times}$. Next localize at $g$ for the sequence above again, we get the following diagram:

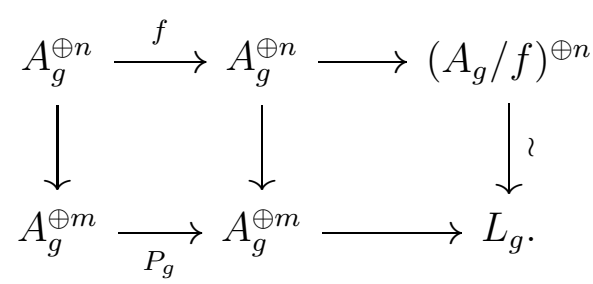


Now taking any prime ideal $\mathfrak{p}$ in Spec $A_{g}$ and localize the diagram above at $\mathfrak{p}$. Then since the top line is a minimal resolution, the vertical morphism as a complex is a split quasi-isomorphism. Therefore we turn out that

$$
\frac{\operatorname{det} P}{f^{n}} \in \underset{\mathfrak{p} \in \operatorname{Spec} A_{g}}{\cap}\left(A_{g}\right)_{\mathfrak{p}}^{\times}=A_{g}^{\times}
$$

Since $f, g$ forms $A$-sequence, we have the equality $A_{f}^{\times} \cap A_{g}^{\times}=A^{\times}$. Therefore we obtain the result.

Now to get a Koszul cube, we are arranging the square above.

Claim 2 There are $n \times m$ matirx $X$ and $n \times n$ matirx $V$ such that $U X=$ $g E_{n}+f V$ where $E_{n}$ is the $n$-th unit matrix.

Proof of Claim 2. For each $k \in(n]$, let us denote one of a pull back of $g \mathfrak{e}_{k}$ in $(A / f)^{\oplus n}$ by the maps

$$
A^{\oplus m} \rightarrow L \hookrightarrow(A / f)^{\oplus n}
$$

by $\mathfrak{x}_{k}=\left(\begin{array}{c}x_{k 1} \\ \vdots \\ x_{k m}\end{array}\right) \in A^{\oplus m}$. Then there is a vector $\mathfrak{v}_{k}=\left(\begin{array}{c}v_{k 1} \\ \vdots \\ v_{k n}\end{array}\right) \in A^{\oplus n}$ such that $U \mathfrak{x}_{k}=g \mathfrak{e}_{k}+f \mathfrak{v}_{k}$ where $\mathfrak{e}_{k}$ is the $k$-th unit vector in $A^{\oplus n}$. We put $X=\left(\begin{array}{lll}\mathfrak{x}_{1} & \cdots & \mathfrak{x}_{n}\end{array}\right)$ and $V=\left(\begin{array}{lll}\mathfrak{v}_{1} & \cdots & \mathfrak{v}_{n}\end{array}\right)$.

Put the matrix $\bar{U}$ as follows:

$$
\bar{U}=\left(\begin{array}{cc}
f V & U \\
X & E_{m}
\end{array}\right)
$$

where $E_{m}$ is the $m$-th elementary matrix. Since we have

$$
\left(\begin{array}{cc}
g E_{n} & 0 \\
0 & E_{m}
\end{array}\right)=\left(\begin{array}{cc}
E_{n} & -U \\
0 & E_{m}
\end{array}\right) \bar{U}\left(\begin{array}{cc}
E_{n} & 0 \\
-X & E_{m}
\end{array}\right)
$$

it follows that $\operatorname{det} \bar{U}=g^{n}$. Now the following diagram is desired $x$ :

$$
\begin{aligned}
& A^{\oplus m+n}--\rightarrow A^{\oplus m+n} \\
& \left(\begin{array}{cc}
E_{n} & 0 \\
0 & P
\end{array}\right) \downarrow \downarrow\left(\begin{array}{cc}
f E_{n} & 0 \\
0 & E_{m}
\end{array}\right) \\
& A^{\oplus m+n} \underset{\bar{U}}{\longrightarrow} A^{\oplus m+n}
\end{aligned}
$$


where dotted map is induced from the commutative diagram below:

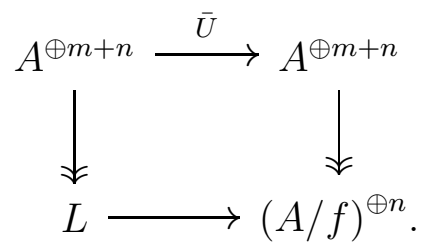

Corollary 7.2. For any $M$ in $\mathbf{W t}_{A}^{(f, g)}$, there are endomorphisms $P, Q$ : $A^{\oplus n} \rightarrow A^{\oplus n}$ such that $M$ is isomorphic to $\frac{A^{\oplus n}}{<\operatorname{Im} P, \operatorname{Im} Q>}$ and $P$ and $Q$ are similar to the following matrixes:

$$
\begin{gathered}
P \sim\left(\begin{array}{cc}
f E_{m} & 0 \\
0 & E_{n-m}
\end{array}\right) \\
Q \sim\left(\begin{array}{cc}
g E_{m} & 0 \\
0 & E_{n-m}
\end{array}\right)
\end{gathered}
$$

where $f, g$ forms a regular sequence.

\section{Application to Gersten conjecture}

In [Ger73] p.28, Gersten proposed the following conjecture

Conjecture 8.1 (Gersten's conjecture). For any commutative regular local ring $A$ and natural numbers $n, p$, the canonical inclusion $\mathcal{M}_{A}^{p+1} \hookrightarrow \mathcal{M}_{A}^{p}$ induces the zero map on $K$-groups

$$
K_{n}\left(\mathcal{M}_{A}^{p+1}\right) \rightarrow K_{n}\left(\mathcal{M}_{A}^{p}\right)
$$

where $\mathcal{M}_{A}^{i}$ is the category of finitely generated $A$-modules $M$ with $\operatorname{Codim}_{\operatorname{Spec} A} \operatorname{Supp} M \geqq i$.

For a regular local ring $A$, in [HM09], we give the following result.

Theorem 8.2 ([HM09] Theorem 5.2.). For a regular ring A, the canonical inclusion functor $\mathbf{W t}_{A}^{p} \hookrightarrow \mathcal{M}_{A}^{p}$ induces an isomorphism on $K$-groups

$$
K\left(\mathbf{W t}_{A}^{p}\right) \stackrel{\simeq}{\rightarrow} K\left(\mathcal{M}_{A}^{p}\right) .
$$


Notations 8.3. For a scheme $X$ and closed subset $Y$, let us denote the category of perfect complexes on $X$ whose cohomological support are in $Y$ by $\operatorname{Perf}_{X}^{Y}$ and put $\operatorname{Perf}_{X}^{p}:=\underset{\operatorname{Codim} Y \geqq p}{\cup} \operatorname{Perf}_{X}^{Y}$.

Rough sketch of proof. In op. cit., we prove that the inclusion functor

$$
\mathbf{C h}^{b}\left(\mathbf{W} \mathbf{t}_{A}^{p}\right) \hookrightarrow \operatorname{Perf}_{A}^{p}
$$

is derived Morita equivalences. But we have the commutative diagram of inclusions below:

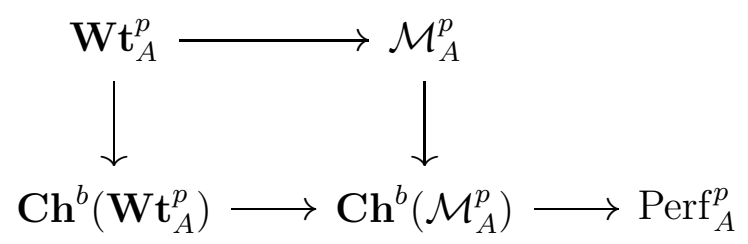

where the vertical inclusions induce isomorphisms on $K$-groups by GilletWaldhausen theorem. So the problem is reduced to that the canonical inclusion $\mathcal{M}_{A}^{p} \hookrightarrow \operatorname{Perf}_{A}^{p}$ induces isomorphisms on $K$-groups. This result is quite well-known. But for the readers convenience, we give a brief proof. We have identities

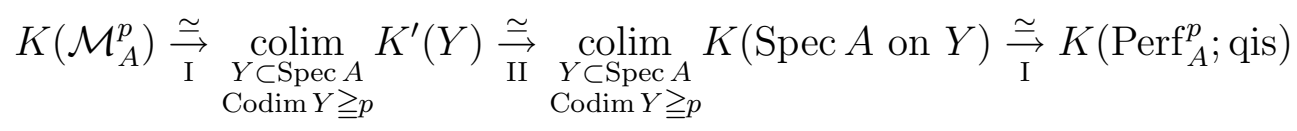

where isomorphisms I are proved by continuity [Qui73], [TT90] and isomorphism II is proved by the Poincaré duality and comparing the following fibration sequences [Qui73] and [TT90]

$$
\begin{gathered}
K^{\prime}(Y) \rightarrow K^{\prime}(\operatorname{Spec} A) \rightarrow K^{\prime}(\operatorname{Spec} A \backslash Y), \\
K(\operatorname{Spec} A \text { on } Y) \rightarrow K(\operatorname{Spec} A) \rightarrow K(\operatorname{Spec} A \backslash Y)
\end{gathered}
$$

for any closed set $Y \subset \operatorname{Spec} A$.

Corollary 8.4. For a regular local ring A, Gersten conjecture is equivalent to the following assertion:

the canonical inclusion $\mathbf{W} \mathbf{t}_{A}^{p+1} \hookrightarrow \mathcal{M}_{A}^{p}$ induces the zero maps on $K$-groups

$$
K_{n}\left(\mathbf{W t}_{A}^{p+1}\right) \rightarrow K_{n}\left(\mathcal{M}_{A}^{p}\right) .
$$


In this paper, we mainly consider $\operatorname{Ker}\left(K_{0}\left(\mathbf{W t}_{A}^{p+1}\right) \rightarrow K_{0}\left(\mathcal{M}_{A}^{p}\right)\right)$ for a Cohen-Macaulay ring $A$.

Example 8.5 (Weight one case). For any $A$ such that every finitely generated projective modules are free, the canonical inclusion $\mathbf{W t}_{A}^{1} \hookrightarrow \mathcal{M}_{A}$ induces the zero map on $K_{0}$-groups.

Proof. Since for every $A$-module $M$ in $\mathbf{W t} t_{A}^{1}$, its projective dimension is one, we have a resolution

$$
0 \rightarrow A^{\oplus m} \rightarrow A^{\oplus n} \rightarrow M \rightarrow 0
$$

There is a non-zero divisor $f$ such that $\operatorname{Supp} M=V(f)$. Now localizing the short exact sequence above by $f$, we turn out that $n=m$. Therefore we have the following equality in $K_{0}\left(\mathcal{M}_{A}\right)$.

$$
[M]=\left[A^{\oplus n}\right]-\left[A^{\oplus n}\right]=0 .
$$

Example 8.6 (Highest weight case). Let $(A, \mathfrak{m})$ be a regular local ring of Krull dimension $d$. Then the canonical inclusion $\mathbf{W} \mathbf{t}_{A}^{d} \hookrightarrow \mathcal{M}_{A}^{d-1}$ induces the zero map on $K_{0}$-groups.

For $A=\mathbb{C}[[x, y, z, w]] /(x y-z w)$, there is an $A$-module $M$ in $\mathbf{W t}_{A}^{3}$ and the class of $M$ in $K_{0}\left(\mathcal{M}_{A}^{2}\right)$ is not zero. (See [Bal07], [DHM85]).

Proof. If $A$ is regular, for each non-negative integer $r, A / \mathfrak{m}^{r}$ is finite projective dimension $A$-Module. Therefore by devissage argument, we turn out that $K_{0}\left(\mathbf{W} \mathbf{t}_{A}^{d}\right)$ is generated by $A / \mathfrak{m}$. Now let $f_{1}, \cdots, f_{d}$ be a regular system of parameter of $A$. Then we have a resolution

$$
0 \rightarrow A /\left(f_{1}, \cdots, f_{d-1}\right) \stackrel{f_{d}}{\rightarrow} A /\left(f_{1}, \cdots, f_{d-1}\right) \rightarrow A / \mathfrak{m} \rightarrow 0 .
$$

Therefore we have the following identity in $K_{0}\left(\mathcal{M}_{A}^{d-1}\right)$.

$$
[A / \mathfrak{m}]=\left[A /\left(f_{1}, \cdots, f_{d-1}\right)\right]-\left[A /\left(f_{1}, \cdots, f_{d-1}\right)\right]=0 .
$$

Therefore the first non-trivial part of this conjecture is weight two case. But as a consequence of previous section, we have the following corollaries. 
Corollary 8.7. Let $A$ be a domain.

(1) For an $A$-sequence $f, g, K_{0}\left(\mathbf{W t}_{A}^{(f, g)}\right) \stackrel{\sim}{\rightarrow} K_{0}\left(\operatorname{Perf}_{A}^{V(f, g)}\right.$; qis) is generated by $\left[\frac{A^{\oplus n}}{<\operatorname{Im} P, \operatorname{Im} Q>}\right]$ where $P$ and $Q$ are endmorphisms on $A^{\oplus n}$ such that $P$ and $Q$ are similar to the following type matrixes:

$$
\begin{gathered}
P \sim\left(\begin{array}{cc}
f^{\alpha} E_{m} & 0 \\
0 & E_{n-m}
\end{array}\right) \\
Q \sim\left(\begin{array}{cc}
g^{\beta} E_{m} & 0 \\
0 & E_{n-m}
\end{array}\right) .
\end{gathered}
$$

(2) If moreover $A$ is a Cohen-Macaulay ring, $K_{0}\left(\mathbf{W t}_{A}^{2}\right) \stackrel{\sim}{\rightarrow} K_{0}\left(\mathrm{Perf}_{A}^{2}\right.$; qis) is generated by $\left[\frac{A^{\oplus n}}{<\operatorname{Im} P, \operatorname{Im} Q>}\right]$ where $P$ and $Q$ are endmorphisms on $A^{\oplus n}$ such that $P$ and $Q$ are similar to the following type matrixes:

$$
\begin{aligned}
& P \sim\left(\begin{array}{cc}
f E_{m} & 0 \\
0 & E_{n-m}
\end{array}\right) \\
& Q \sim\left(\begin{array}{cc}
g E_{m} & 0 \\
0 & E_{n-m}
\end{array}\right)
\end{aligned}
$$

where $f$ and $g$ forms A-sequences.

(3) Moreovere if $A$ is regular noetherian, $K_{0}\left(\mathcal{M}_{A}^{2}\right)$ is generated by the modules in (2).

(4) Moreover, if $P$ and $Q$ are commutative, $\left[\frac{A^{\oplus n}}{<\operatorname{Im} P, \operatorname{Im} Q>}\right]$ is in $\operatorname{Ker}\left(K_{0}\left(\mathbf{W t}_{A}^{2}\right) \rightarrow\right.$ $\left.K_{0}\left(\mathcal{M}_{A}^{1}\right)\right)$.

Proof. (1), (2) and (3) are direct corollaries of previous section and [HM09]. Now assume that $P$ and $Q$ are commutative. Then

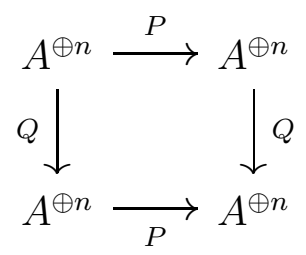

is a Koszul cube resolution of $\frac{A^{\oplus n}}{\langle\operatorname{Im} P, \operatorname{Im} Q>}$. (We call such a Koszul cube as commutative.) Therefore we have the following short exact sequence

$$
0 \rightarrow A^{\oplus n} / \operatorname{Im} P \stackrel{\bar{Q}}{\rightarrow} A^{\oplus n} / \operatorname{Im} P \rightarrow \frac{A^{\oplus n}}{<\operatorname{Im} P, \operatorname{Im} Q>} \rightarrow 0 .
$$


Therefore we have the following identity in $K_{0}\left(\mathcal{M}_{A}^{1}\right)$.

$$
\left[\frac{A^{\oplus n}}{<\operatorname{Im} P, \operatorname{Im} Q>}\right]=\left[A^{\oplus n} / \operatorname{Im} P\right]-\left[A^{\oplus n} / \operatorname{Im} P\right]=0 .
$$

Corollary 8.8. For a 3-dimensional regular local ring, Gersten's conjecture for $K_{0}$-groups is true if and only if $K_{0}\left(\mathbf{W t}^{2}(A)\right)$ is generated by the class of modules $\left[\frac{A^{\oplus n}}{<\operatorname{Im} P, \operatorname{Im} Q>}\right]$ where $P$ and $Q$ are commutative.

Proof. It is just a direct corollary of 1.2, 8.4, 8.5, 8.6 and 8.7.

The second author propose the following conjecture.

Conjecture 8.9. For any two dimensional Koszul cube $x$, there is a commutative two dimensional Koszul cube $y$ and a morphism of cubes $f: x \rightarrow y$ such that it forms three dimensional Koszul cubes.

Proposition 8.10. The conjecture 8.9 implies Gersten's conjecture for $K_{0^{-}}$ groups for a three dimensional regular local ring.

Proof. It is enough to show that $\pi_{0}^{2}$ is zero map by 8.5 and 8.6.

For any weight two module $M$, there exist a Koszul cube resolution $x$ of $M$ by 7.1 . Then by the conjecture 8.9 , there exist a commutative Koszul cube $y$ and a map $x \stackrel{f}{\rightarrow} y$ such that $z:=[x \stackrel{f}{\rightarrow} y]$ is a three dimensional Koszul cube. Then we have a short exact sequence:

$$
0 \rightarrow M \rightarrow \mathrm{H}_{0}(\operatorname{Tot}(y)) \rightarrow \mathrm{H}_{0}(\operatorname{Tot}(z)) \rightarrow 0
$$

By 8.6, the class of $\mathrm{H}_{0}(\operatorname{Tot}(z))$ is zero in $K_{0}\left(\mathcal{M}_{A}^{2}\right)$. Therefore in $K_{0}\left(\mathcal{M}_{A}^{1}\right)$ we have an equalities:

$$
[M]=\left[\mathrm{H}_{0}(\operatorname{Tot}(z))\right]=0
$$

where the last equality coming from 8.7.

\section{References}

[Bal07] P. Balmer, Niveau spectral sequences on singular schemes and failure of generalized Gersten conjecture, Proc. Amer. Math. Soc. 137, (2009), 99-106. 
[BE73] D. A. Buchsbaum and D. Eisenbud, What makes a complex exact?, J. Algebra 25 (1973), p.259-268.

[DHM85] S. P. Dutta, M. Hochster and J. E. McLaughlin, Modules of finite projective dimension with negative intersection multiplicities, Invent. Math. 79 (1985), p.253-291.

[Ger73] S. Gersten, Some exact sequences in the higher K-theory of rings, In Higher K-theories, Springer Lect. Notes Math. 341 (1973), p.211243.

[Ger74] S. Gersten, The localization theorem for projective modules, Comm. Alg. 2 (1974), p.307-350.

[HM09] T. Hiranouchi and S. Mochizuki, Pure weight perfect Modules on divisorial schemes, to appear in proceedings of "Algebraic and Geometric Deformation Spaces" (2009).

[Kos] S. Mochizuki, Higher K-theory of Koszul cubes, available at http://www.math.uiuc.edu/K-theory/0895/ (2008).

[Lev85] M. Levine, A K-theoretic approach to multiplicities, Math. Ann. 271 (1985), p.451-458.

[Qui73] D. Quillen, Higher algebraic K-theory I, In Higher K-theories, Springer Lect. Notes Math. 341 (1973), p.85-147.

[TT90] R. W. Thomason and T. Trobaugh, Higher K-theory of schemes and of derived categories, In The Grothendieck Festscrift, Vol III, (1990), p.247-435.

[Wei94] C. A. Weibel, An introduction to homological algebra, Cambridge studies in advanced mathematics 38, (1994). 